\title{
Protected cytoskeletal-related proteins: Towards a resolution of contradictions regarding the role of the cytoskeleton in cancer
}

\author{
DANIEL T. SEGARRA ${ }^{1}$, JOHN M. YAVORSKI ${ }^{1}$ and GEORGE BLANCK ${ }^{1,2}$ \\ ${ }^{1}$ Department of Molecular Medicine, Morsani College of Medicine, University of South Florida; \\ ${ }^{2}$ H. Lee Moffitt Cancer Center and Research Institute, Tampa, FL 33612, USA
}

Received February 8, 2017; Accepted March 20, 2017

DOI: $10.3892 /$ br.2017.940

\begin{abstract}
Initial reports of the role of the cytoskeleton in cancer indicated that tumor cells with a more disorganized cytoskeleton were more tumorigenic. These reports were based on stains for the F-actin cytoskeleton, for example, using phalloidin or anti-F-actin antibody reagents, and gave a basic impression of F-actin-based cytoskeletal integrity. Later developments emphasized the significance of the cytoskeletal elements in cell migration, presumably associated with either basement membrane invasion or metastasis, or both, with several specific proteins implicated in the formation of cell invadopodia. With the advent of genomics approaches, it has become clear that cytoskeletal related proteins are indeed common targets of mutagenesis in cancer and commonly rank among the most mutated proteins in cancers, presumably due to large coding region sizes and the significant stochastic component to human mutagenesis. This cytoskeletal genomics result is consistent with the loss of cytoskeleton integrity as a hallmark of tumor development, but raises the question of whether such mutational sensitivity relates to the migration and invadopodia aspects of tumor progression. In the present study, the authors report that it is possible to identify a set of cytoskeletal related proteins protected from mutation, in comparison to the commonly mutated cytoskeleton related proteins in certain, but not all cancer, datasets.
\end{abstract}

\section{Introduction}

The tumor cell cytoskeleton first became an important subject in cancer biology when attempts were made to isolate tumor revertants by cloning slowly dividing cells resistant to chemicals that could be incorporated into DNA and poison rapidly dividing cells $(1,2)$. Initial observations indicated that revertants were flat, i.e., well spread in a tissue culture

Correspondence to: Dr George Blanck, Department of Molecular Medicine, Morsani College of Medicine, University of South Florida, 12901 Bruce B. Downs Boulevard, Tampa, FL 33612, USA E-mail: gblanck@health.usf.edu

Key words: The Cancer Genome Atlas, cytoskeletal and extra-cellular matrix proteins, mutations, metastasis, melanoma, bladder cancer dish, which led to considerations of 'flat revertants' having an extensive cytoskeletal architecture. This indeed turned out to be verifiable with a variety of stains for the F-actin cytoskeleton $(3,4)$. These results in turn led to an extensive body of work indicating that cells with a more disorganized cytoskeleton were more tumorigenic, particularly as determined by in vitro parameters corresponding to a malignant phenotype (5), such as growth in soft agar; as determined by experiments with immune-compromised mice (6). More recently, genetic techniques have implicated specific, cytoskeletal dysfunctions as favoring tumorigenesis, for example, mouse tumors engineered to lack dystrophin $(7,8)$, which links to the cytoskeleton, are more aggressive.In addition, The Cancer Genome Atlas (TCGA) data have revealed that cytoskeletal protein-related coding regions (CPCRs), including extracellular matrix protein coding regions, are among the most frequently mutated coding regions in cancer $(9,10)$, and these CPCR mutations have been indicated as driver mutations in certain types of cancers (11).

However, other study has indicated that tumor cell migration and tumor cell invasive properties are dependent on a functioning actin cytoskeleton and the function of cytoskeleton-dependent invadopodia (12-17). Several cytoskeletal related proteins in particular have been implicated in cell migration and invadopodia. For example, vinculin has been demonstrated to be important for polarized cell motility and metastasis $(18,19)$; and talin-1 has been demonstrated to be important in tissue extravasion and has been considered as a target to prevent metastasis (20). On the other hand, both of these proteins have been considered as potential tumor suppressor proteins, with knock-out approaches contradicting the idea that these two proteins are important in their wild-type forms for cell migration or metastasis $(21,22)$.

To help resolve the above cytoskeleton-related contradiction, we decided to test the hypothesis that mutation rates could define two sets of CPCRs: a CPCR-mutated set, as previously described (9-11); and a CPCR-protected set, with a candidate CPCR-protected set identified below for several TCGA cancer data sets.

\section{Materials and methods}

Mutated cytoskeletal gene expression, mutated set, protected set. The TCGA data portal (http://cancergenome.nih.gov. 


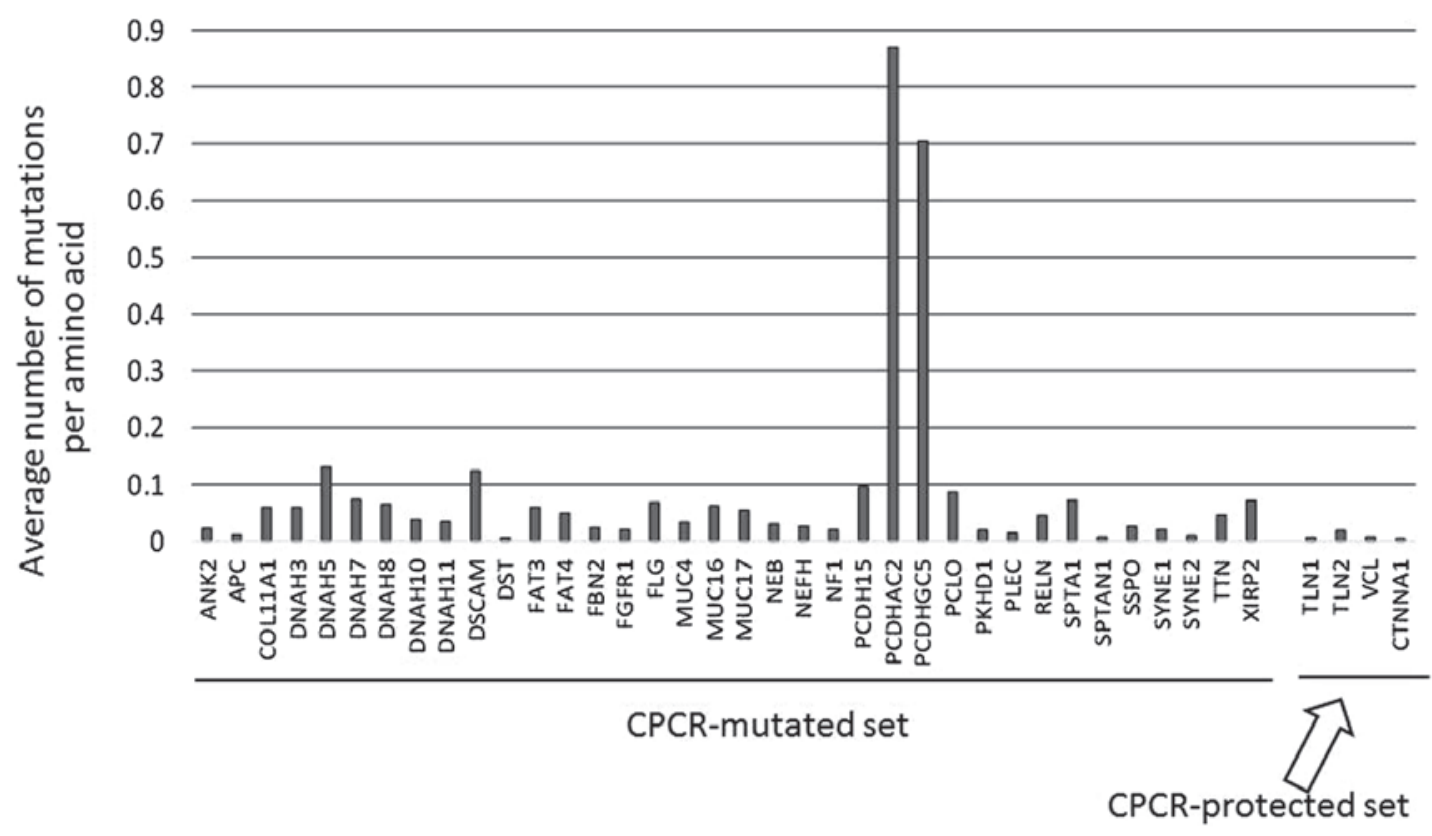

Figure 1. Average number of mutations in metastatic SKCM for each indicated cytoskeletal protein, normalized to amino acid length $(\mathrm{P}<0.012)$. The CPCR-mutated set is from ANK2 to XIRP2. The CPCR-protected set is the last four coding regions. SKCM, skin cutaneous melanoma; CPCR, cytoskeletal protein-related coding regions.

ezproxy.lib.usf.edu/), under NIH/dbGAP project approval no. 6300, was used to collect somatic mutation data for bladder urothelial carcinoma (BLCA), breast invasive carcinoma (BRCA), cervical squamous cell carcinoma and endocervical adenocarcinoma (CESC), head and neck squamous cell carcinoma (HNSC), liver hepatocellular carcinoma (LIHC), prostate adenocarcinoma (PRAD), rectum adenocarcinoma (READ), skin cutaneous melanoma-primary (SKCM-01), skin cutaneous melanoma-metastatic (SKCM-06), and stomach adenocarcinoma (STAD) data sets. (These data are now available from the cBioPortal (http://www.cbioportal. org/index.do). Tumor sample barcodes in the somatic mutation (exome) files were truncated to contain only the following characters, TCGA-\#\#-\#\#\#\#. Mutation data from the comprehensive mutation files were collected for the CPCR sets (HUGO symbols listed in Fig. 1) and a Microsoft Excel COUNTIF function was used to determine the number of mutations for each CPCR. Total mutations were then normalized to amino acid length for respective coding regions.

Deleterious amino acid changes: PROVEAN.The chromosome number, start position, reference allele and tumor sequence allele data were collected for each of the datasets. These data were then copied into PROVEAN under the 'Human Genome Variants' protocol. The removal of duplicates from the 'INPUT' column in the PROVEAN output was used to determine the number of deleterious amino acid changes for each dataset. A Microsoft IF[ISERROR(MATCH)] function was then used to separate all mutations pertaining to the protected and mutated CPCR sets. The number of deleterious amino acid changes was then divided by the sample size to determine the average number of deleterious amino acid changes per barcode for each tumor set. BLCA data was further divided into deleterious mutations belonging to No Subsequent Tumor or New Tumor groups based on data from TCGA clinical files, as described previously (23).

Supporting online material. The original data and the details of the calculations in this report are available at http://www. universityseminarassociates.com/Supporting_online_material_for_scholarly_pubs.php.

\section{Results and Discussion}

To obtain an indication of whether mutation frequencies were more prevalent in a previously defined, heavily mutated set of CPCRs-mutated (9-11), than in a set of cytoskeletal protein related coding regions associated with pro-tumorigenic properties (CPCR-protected; see Materials and methods), the authors performed the following processing steps: Genome sequencing files from TCGA, representing 10 distinct cancer datasets (CESC, BRCA, STAD, PRAD, HNSC, LIHC, READ, BLCA, SKCM-primary and SKCM-metastatic), were downloaded, and total mutations within the above gene sets were tabulated. The mutation counts for each coding region were normalized to amino acid length (Fig. 1). Results indicated that the two gene groups, CPCR-mutated and CPCR-protected had significantly different mutation rates for READ, PRAD, HNSC, LIHC, BLCA, SKCM-primary, and SKCM-metastasis datasets, but not for the CESC, BRCA, or STAD datasets (Fig. 2).

Mutation data was then analyzed using the PROVEAN web tool, using the human genome variants protocol of PROVEAN. The output was sorted based on the PROVEAN prediction of deleterious or neutral. Fig. 3 demonstrates the example of SKCM-metastatic, which demonstrated significant difference between the two CPCR sets $(\mathrm{P}<0.00035)$, with fewer deleterious mutations in the CPCR-protected set. A total of four of the ten cancer datasets (READ, BLCA, SKCM-primary and 


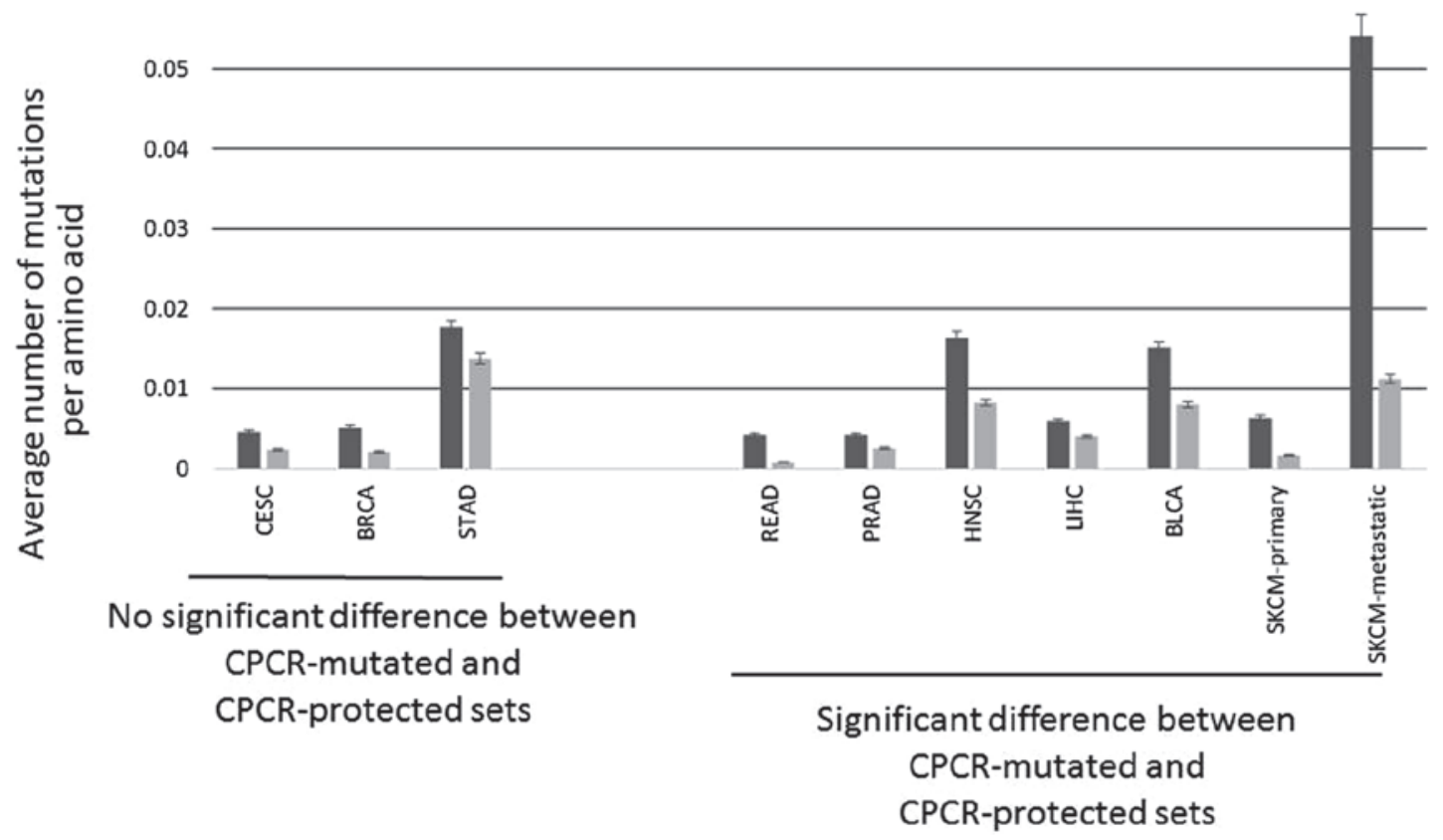

Figure 2. Average number of total mutations for the indicated cancer datasets, for the CPCR-mutated (black) and -protected (grey) sets, normalized to amino acid lengths. Data are presented as the mean \pm standard deviation. (READ P $<0.01$, PRAD P $<0.009$, HNSC P $<8.12$ E-06, LIHC P $<0.003$, BLCA P $<0.001$, SKCM-primary $\mathrm{P}<0.015$, SKCM-metastatic $\mathrm{P}<0.012$; CESC, BRCA, STAD, left side of figure, not significant). CPCR, cytoskeletal protein-related coding regions; READ, rectum adenocarcinoma; PRAD, prostate adenocarcinoma; HNSC, head and neck squamous cell carcinoma; LIHC, liver hepatocellular carcinoma; BLCA, bladder urothelial carcinoma; SKCM, skin cutaneous melanoma; CESC, cervical squamous cell carcinoma and endocervical adenocarcinoma; BRCA, breast invasive carcinoma; STAD, stomach adenocarcinoma.

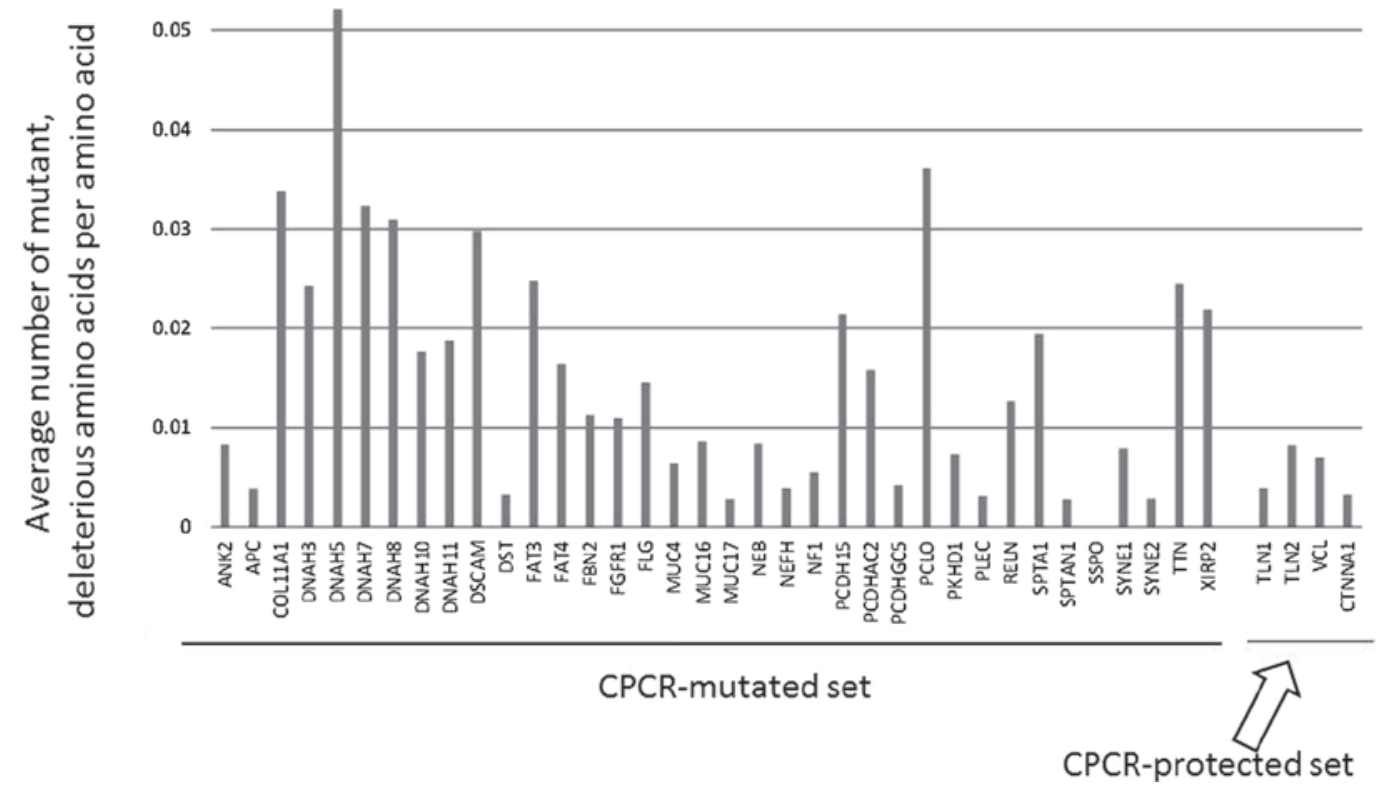

Figure 3. Average number of cytoskeletal protein deleterious amino acid substitutions in metastatic SKCM samples, for the CPCR-mutated and -protected sets, normalized to amino acid lengths $(\mathrm{P}<0.00035)$. The CPCR-mutated set is from ANK2 to XIRP2. The CPCR-protected set is the last four coding regions. SKCM, skin cutaneous melanoma; CPCR, cytoskeletal protein-related coding regions.

SKCM-metastatic) also demonstrated significant differences in average total deleterious mutations between CPCR-mutated and CPCR-protected sets (Fig. 4). In other words, two of the cancer datasets above, PRAD and HNSC, which indicated a statistically significant difference between the mutation rates for the CPCR-protected vs. CPCR-mutated sets did not maintain that distinction using the standard of deleterious mutations, presumably a more stringent standard.
To learn whether the CPCR-protected set could provide a further indication of fewer mutations being important for disease progression, the number of mutations and deleterious amino acids per barcode in the SKCM-primary and SKCM-metastasis datasets was determined. Results indicated that the CPCR-mutated set and the CPCR-protected set had similar increases in mutations and deleterious amino acids going from primary to metastatic samples (Table I). 
Table I. Comparison of mutations per barcode for the CPCR-protected sets, for primary and metastatic SKCM.

CPCR-mutated set

\begin{tabular}{|c|c|c|c|c|c|}
\hline Tumors & No. of mutations & $\begin{array}{l}\text { No. of deleterious } \\
\text { AA substitutions }\end{array}$ & $\begin{array}{c}\text { Total } \\
\text { barcodes }\end{array}$ & $\begin{array}{l}\text { Mutations } \\
\text { per barcode }\end{array}$ & $\begin{array}{l}\text { Deleterious AA substitutions } \\
\text { per barcode }\end{array}$ \\
\hline SKCM-primary & 1,182 & 338 & 63 & 18.76 & 5.36 \\
\hline SKCM-metastatic & 10,055 & 2,948 & 278 & 36.16 & 10.60 \\
\hline
\end{tabular}

CPCR-protected set

\begin{tabular}{lccccc}
\hline Tumors & No. of mutations & $\begin{array}{c}\text { No. of deleterious } \\
\text { AA substitutions }\end{array}$ & $\begin{array}{c}\text { Total } \\
\text { barcodes }\end{array}$ & $\begin{array}{c}\text { Mutations } \\
\text { per barcode }\end{array}$ & $\begin{array}{c}\text { Deleterious AA substitutions } \\
\text { per barcode }\end{array}$ \\
\hline SKCM-primary & 12 & 4 & 63 & 0.190 & 0.063 \\
SKCM-metastatic & 80 & 42 & 278 & 0.287 & 0.151 \\
\hline
\end{tabular}

CPCR, cytoskeletal protein-related coding regions; SKCM, skin cutaneous melanoma.

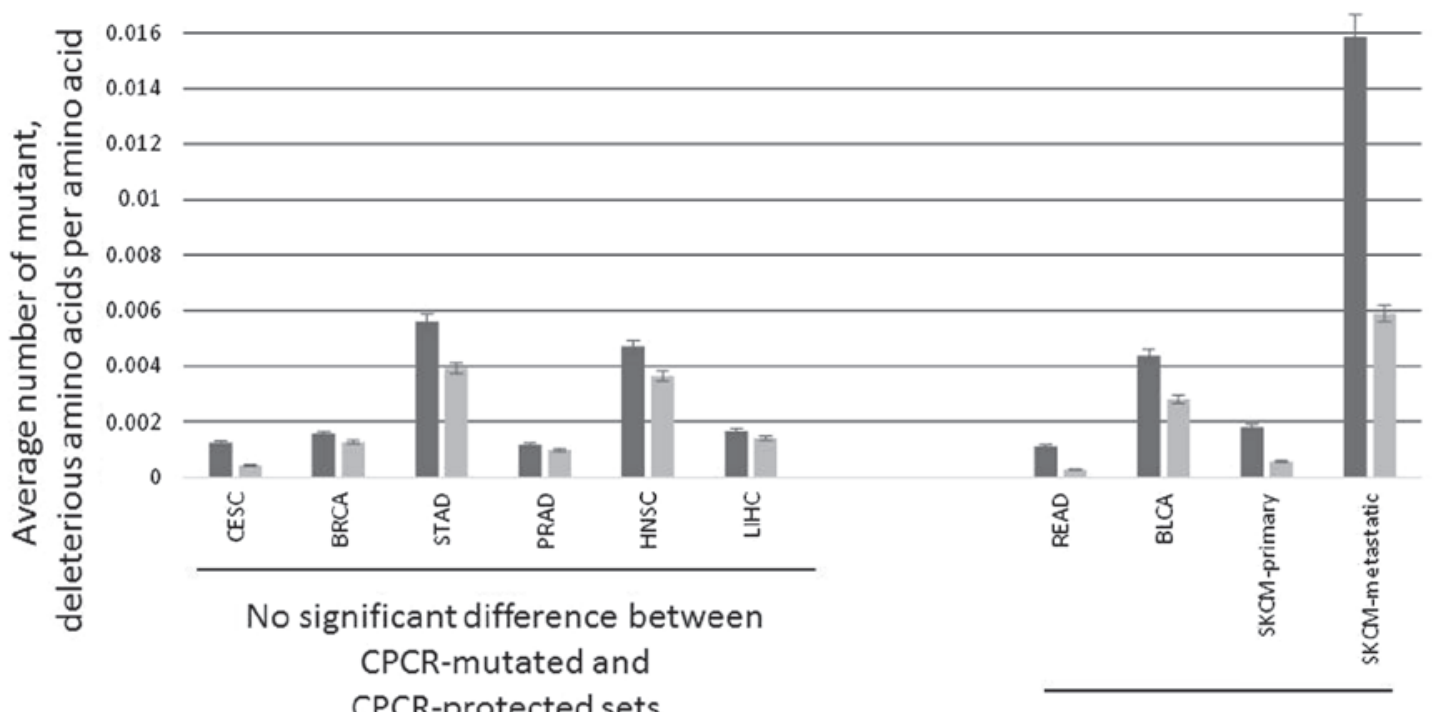

Significant difference between
CPCR-mutated and
CPCR-protected sets

Figure 4. Average total deleterious amino acid substitutions for the indicated cancer datasets, normalized to amino acid length (READ P $<0.036$, BLCA $\mathrm{P}<0.006$, SKCM-primary P<0.0086, SKCM-06 P<0.00035; CESC, BRCA, STAD, PRAD, HNSC, LIHC, left side of figure, not significant). READ, rectum adenocarcinoma; BLCA, bladder urothelial carcinoma; SKCM, skin cutaneous melanoma; CESC, cervical squamous cell carcinoma and endocervical adenocarcinoma; BRCA, breast invasive carcinoma; STAD, stomach adenocarcinoma; PRAD, prostate adenocarcinoma; HNSC, head and neck squamous cell carcinoma; LIHC, liver hepatocellular carcinoma.

The authors further pursued this question by analyzing two sets of BCLA barcodes available via TCGA: BLCA barcodes where there was no known subsequent tumor and barcodes where a new tumor was known, as detailed previously (23). Again, there was no difference in the increase in the rate of deleterious amino acids for the two sets of CPCRs, when no subsequent tumor and new tumor were compared (Table II). To address this issue a final time, the mutations per barcode were assessed for the two different CPCR sets for PRAD-primary vs. two sets of PRAD-metastatic barcodes, and again, there was no increase in one of the CPCR sets that was statistically, significantly greater or less than the other set (Table III). In the case of PRAD, it needs to be kept in mind that there was no evidence of a difference in the CPCR-mutated vs. CPCR-protected set for the deleterious amino acid replacements. The PRAD distinction for these two CPCR sets was limited to the mutations rates without regard to amino acid changes (Fig. 2).

The above data strongly support the idea that CPCRs can be divided into sets with statistically different rates of mutation, 
Table II. Comparison of BLCA deleterious AA substitutions per barcode between CPCR-mutated and CPCR-protected sets for New tumor and No subsequent tumor barcodes.

CPCR-mutated set

\begin{tabular}{lccccc}
\hline Tumors & No. of mutations & $\begin{array}{c}\text { No. of deleterious } \\
\text { AA substitutions }\end{array}$ & $\begin{array}{c}\text { Total } \\
\text { barcodes }\end{array}$ & $\begin{array}{c}\text { Mutations } \\
\text { per barcode }\end{array}$ & $\begin{array}{c}\text { Deleterious AA substitutions } \\
\text { per barcode }\end{array}$ \\
\hline No subsequent tumor & 217 & 66 & 27 & 8.03 & 2.44 \\
New tumor & 195 & 55 & 28 & 6.96 & 1.96 \\
\hline
\end{tabular}

CPCR-protected set

\begin{tabular}{lccccc}
\hline Tumors & No. of mutations & $\begin{array}{c}\text { No. of deleterious } \\
\text { AA substitutions }\end{array}$ & $\begin{array}{c}\text { Total } \\
\text { barcodes }\end{array}$ & $\begin{array}{c}\text { Mutations } \\
\text { per barcode }\end{array}$ & $\begin{array}{c}\text { Deleterious AA substitutions } \\
\text { per barcode }\end{array}$ \\
\hline No subsequent tumor & 4 & 1 & 27 & 0.148 & 0.037 \\
New tumor & 3 & 1 & 28 & 0.107 & 0.035 \\
\hline
\end{tabular}

BLCA, bladder urothelial carcinoma; CPCR, cytoskeletal protein-related coding regions.

Table III. Comparison of mutations per barcode between CPCR-mutated and CPCR-protected sets, for primary and metastatic PRAD.

CPCR-mutated set

\begin{tabular}{lccc}
\hline Tumors & No. of mutations & Total barcodes & Mutations per barcode \\
\hline PRAD-primary & 784 & 499 & 1.57 \\
PRAD-metastatic & 77 & 61 & 1.26 \\
Prostate-metastatic & 373 & 150 & 2.48 \\
\hline
\end{tabular}

CPCR-protected set

\begin{tabular}{lccc}
\hline Tumors & No. of mutations & Total barcodes & Mutations per barcode \\
\hline PRAD-primary & 18 & 499 & 0.036 \\
PRAD-metastatic & 1 & 61 & 0.016 \\
Prostate-metastatic & 13 & 150 & 0.086 \\
\hline
\end{tabular}

CPCR, cytoskeletal protein-related coding regions; PRAD, prostate adenocarcinoma.

including mutations that lead to deleterious amino acid substitutions. This conclusion may serve a role in resolving the contradictions in the literature that indicate that cytoskeletal disorganization is a hallmark of tumorigenesis but is also an important feature of cell migration, and presumably a feature of metastatic cells. However, the above data do not indicate that these distinctions are consistent with a change in tumor aggressiveness. While this is a negative result, and cannot lead to a final conclusion, the current data are consistent with the possibility that CPCR-mutated and CPCR-protected sets are a fundamental aspect of the generation of the tumor cell, rather than representative of a distinction between aggressive and non-aggressive cancers.

\section{References}

1. Vogel A, Risser R and Pollack R: Isolation and characterization of revertant cell lines. 3. Isolation of density-revertants of SV40-transformed 3T3 cells using colchicine. J Cell Physiol 82: 181-188, 1973.

2. Pollack RE, Green H and Todaro GJ: Growth control in cultured cells: Selection of sublines with increased sensitivity to contact inhibition and decreased tumor-producing ability. Proc Natl Acad Sci USA 60: 126-133, 1968.

3. Verderame M, Alcorta D, Egnor M, Smith K and Pollack R: Cytoskeletal F-actin patterns quantitated with fluorescein isothiocyanate-phalloidin in normal and transformed cells. Proc Natl Acad Sci USA 77: 6624-6628, 1980.

4. Kopelovich L, Conlon S and Pollack R: Defective organization of actin in cultured skin fibroblasts from patients with inherited adenocarcinoma. Proc Natl Acad Sci U S A 74: 3019-3022, 1977. 
5. Shin SI, Freedman VH, Risser R and Pollack R: Tumorigenicity of virus-transformed cells in nude mice is correlated specifically with anchorage independent growth in vitro. Proc Natl Acad Sci USA 72: 4435-4439, 1975.

6. Steinberg BM, Rifkin D, Shin S, Boone C and Pollack R: Tumorigenicity of revertant from an SV40-transformed line. J Supramol Struct 11: 539-546, 1979.

7. Wang Y, Marino-Enriquez A, Bennett RR, Zhu M, Shen Y, Eilers G, Lee JC, Henze J, Fletcher BS, Gu Z, et al: Dystrophin is a tumor suppressor in human cancers with myogenic programs. Nat Genet 46: 601-606, 2014.

8. Fernandez K, Serinagaoglu Y, Hammond S, Martin LT and Martin PT: Mice lacking dystrophin or alpha sarcoglycan spontaneously develop embryonal rhabdomyosarcoma with cancer-associated p53 mutations and alternatively spliced or mutant Mdm2 transcripts. Am J Pathol 176: 416-434, 2010.

9. Parry ML, Ramsamooj M and Blanck G: Big genes are big mutagen targets: A connection to cancerous, spherical cells? Cancer Lett 356 (2 Pt B): 479-482, 2015.

10. Parry ML and Blanck G: Flat cells come full sphere: Are mutant cytoskeletal-related proteins oncoprotein-monsters or useful immunogens? Hum Vaccin Immunother 12: 120-123, 2016.

11. Fawcett TJ, Parry ML and Blanck G: A Novel Approach to Evaluating Cancer Driver Gene Mutation Densities: Cytoskeleton-related Gene Candidates. Cancer Genomics Proteomics 12: 283-290, 2015.

12. Zachary JM, Cleveland G, Kwock L, Lawrence T, Weissman RM, Nabell L, Fried FA, Staab EV, Risinger MA and Lin S: Actin filament organization of the Dunning R3327 rat prostatic adenocarcinoma system: Correlation with metastatic potential. Cancer Res 46: 926-932, 1986.

13. Xu W, Mezencev R, Kim B, Wang L, McDonald J and Sulchek T: Cell stiffness is a biomarker of the metastatic potential of ovarian cancer cells. PLoS One 7: e46609, 2012.

14. Bear JE and Haugh JM: Directed migration of mesenchymal cells: Where signaling and the cytoskeleton meet. Curr Opin Cell Biol 30: 74-82, 2014.
15. Verschueren H, Van der Taelen I, Dewit J, De Braekeleer J and De Baetselier P: Metastatic competence of BW5147 T-lymphoma cell lines is correlated with in vitro invasiveness, motility and F-actin content. J Leukoc Biol 55: 552-556, 1994.

16. Nürnberg A, Kitzing T and Grosse R: Nucleating actin for invasion. Nat Rev Cancer 11: 177-187, 2011.

17. Kim TY, Vigil D, Der CJ and Juliano RL: Role of DLC-1, a tumor suppressor protein with RhoGAP activity, in regulation of the cytoskeleton and cell motility. Cancer Metastasis Rev 28: 77-83, 2009.

18. Carisey A, Tsang R, Greiner AM, Nijenhuis N, Heath N, Nazgiewicz A, Kemkemer R, Derby B, Spatz J and Ballestrem C: Vinculin regulates the recruitment and release of core focal adhesion proteins in a force-dependent manner. Curr Biol 23: 271-281, 2013.

19. Thakur RK, Yadav VK, Kumar A, Singh A, Pal K, Hoeppner L, Saha D, Purohit G, Basundra R, Kar A, et al: Non-metastatic 2 (NME2)-mediated suppression of lung cancer metastasis involves transcriptional regulation of key cell adhesion factor vinculin. Nucleic Acids Res 42: 11589-11600, 2014.

20. Gilardi M, Bersini S, Calleja AB, Kamm RD, Vanoni M and Moretti M: PO-12 - The key role of talin-1 in cancer cell extravasation dissected through human vascularized 3D microfluidic model. Thromb Res 140 (Suppl 1): S180-S181, 2016.

21. Liu M, Oberg K and Zhou Y: Expression and function of vinculin in neuroendocrine tumors. Tumour Biol 28: 196-204, 2007.

22. Zhang W, Mao YQ, Wang H, Yin WJ, Zhu SX and Wang WC: MiR-124 suppresses cell motility and adhesion by targeting talin 1 in prostate cancer cells. Cancer Cell Int 15: 49, 2015.

23. Samy MD, Tong WL, Yavorski JM, Sexton WJ and Blanck G $\mathrm{T}$ cell receptor gene recombinations in human tumor specimen exome files: Detection of T cell receptor- $\beta$ VDJ recombinations associates with a favorable oncologic outcome for bladder cancer. Cancer Immunol Immunother 66: 403-410, 2017. 